\title{
TLR9 activation cooperates with T cell checkpoint blockade to regress poorly immunogenic melanoma
}

\author{
Matthew J. Reilley ${ }^{1 \dagger}$, Brittany Morrow ${ }^{2 \dagger}$, Casey R. Ager ${ }^{2,4}$, Arthur Liu ${ }^{2,4}$, David S. Hong ${ }^{3}$ and Michael A. Curran ${ }^{2,4^{*}}$ (D)
}

\begin{abstract}
Tumors that lack pre-existing immune infiltration respond poorly to $T$ cell checkpoint blockade immunotherapy. These cancers often surround themselves with high densities of suppressive myeloid stroma while excluding immunostimulatory dendritic cells. Tumor-resident myeloid cells and selected lymphocyte populations retain expression of Toll-like Receptors (TLR) that sense common features of pathogens and activate innate immunity in response. We explored whether agonists of TLR9 could augment innate immunity to promote tumor regression alone or in combination with T cell checkpoint blockade. In the setting of the immunogenic B16-Ova (Ovalbumin) expressing melanoma model, local injection of the CpG oligonucleotide TLR9 agonist ODN1826 combined with systemic CTLA-4 blockade cured $45 \%$ of mice of both their treated and an untreated tumor on the opposite flank demonstrating the synergistic potential of this combination. Next, in the non-immunogenic B16-F10 melanoma model, we showed that only intra-tumoral, but not systemic TLR9 activation augments the therapeutic potential of checkpoint blockade. In this setting, intra-tumoral TLR9 activation cooperated equally with either CTLA-4 or PD-1 blockade co-administered locally or given systemically; however, the uninjected tumor rarely regressed. Anti-CTLA-4 combinations were associated with improved intra-tumoral CD8 to regulatory $T$ cell ratios, while anti-PD-1 combinations elicited improved ratios of CD8 T cells relative to suppressive myeloid stroma. Using both a TLR9 agonist (MGN1703) and a CTLA-4 antibody (9D9-IgG2a) of increased potency cured 50\% of bi-lateral B16-F10 melanoma. These findings suggest that intra-tumoral TLR9 agonists can improve sensitivity of poorly immunogenic tumors to T cell checkpoint blockade, and that newer, higher potency TLR agonists and checkpoint antibodies can raise the therapeutic ceiling for this combination therapy.
\end{abstract}

Keywords: TLR9, CTLA-4, PD-1, Immunotherapy, MGN1703

\section{Introduction}

Tumors actively condition their microenvironments to foster recruitment of suppressive myeloid stroma and dampen accumulation of potentially immunostimulatory antigen-presenting cells such as dendritic cells. Lack of pro-inflammatory myeloid cells fosters immune ignorance of the tumor as a result of insufficient tumor antigen cross-presentation. Further, the predominant M2 macrophage and myeloid-derived suppressor cell

\footnotetext{
* Correspondence: mcurran@mdanderson.org

${ }^{\dagger}$ Matthew J. Reilley and Brittany Morrow contributed equally to this work.

${ }^{2}$ Department of Immunology, The University of Texas MD Anderson Cancer

Center, Houston, TX 77030, USA

${ }^{4}$ The University of Texas MD Anderson UTHealth Graduate School of Biomedical Sciences; Immunology Program, Houston, TX 77030, USA Full list of author information is available at the end of the article
}

(MDSC) composition of the myeloid stroma effectively shields the tumor from any adaptive immune effectors which do become mobilized. In this setting, blockade of $\mathrm{T}$ cell immune checkpoint receptors is often insufficient to mediate any significant regression of cancer.

Toll-like receptors (TLR) sense common features of pathogens and, in response, trigger innate immune activation including secretion of type I Interferons. Provision of toll-like receptor ligands has the potential to reactivate tumor stroma, particularly myeloid cells and $\mathrm{B}$ cells, thus increasing both tumor antigen crosspresentation and pro-inflammatory cytokine production [1]. These direct effects on innate immune activation, in turn, foster enhanced activation of adaptive immune effectors (i.e. $\mathrm{T}$ and NK cells) increasing both baseline

(c) The Author(s). 2019 Open Access This article is distributed under the terms of the Creative Commons Attribution 4.0 International License (http://creativecommons.org/licenses/by/4.0/), which permits unrestricted use, distribution, and 
tumor immune infiltration as well as sensitivity to $\mathrm{T}$ cell checkpoint blockade therapy.

Agonists of Toll-like receptor 9 (TLR9), which recognizes DNA with unmethylated CpG motifs, can activate B cells, myeloid dendritic cells, and plasmacytoid dendritic cells [2]. Prior publications have demonstrated the potential of various TLR9 agonists administered via intra-tumor injection to augment anti-tumor immunity alone or in combination with $\mathrm{T}$ cell checkpoint blocking or $\mathrm{T}$ cell costimulatory agonist antibodies [3-8]. Despite this, the optimal route of administration for TLR9 agonists, as well as their compatibility with current FDA-approved checkpoint blockade antibodies remains unknown. Further, synthetic TLR9 agonists with enhanced potency relative to classical oligodeoxynucleotide (ODN) agonists have been developed; however, whether the in vitro potency of these drugs translates to enhanced in vivo immunotherapeutic potential has yet to be determined.

Here we show that intra-tumoral administration of the TLR9 agonist ODN1826 [9] synergizes with CTLA-4 blockade to promote rejection of bi-laterally implanted B16-Ovalbumin (B16-Ova) melanoma. As innate agonists of both TLR and the Stimulator of Interferon Genes pathways are now being administered to patients both intratumorally as well as systemically, we investigated the impact of route of delivery on the efficacy of ODN1826 with or without anti-CTLA-4 or anti-PD-1 on the progression of bi-laterally implanted B16-F10 parental melanoma. While intra-tumoral ODN1826 benefitted from being combined with either CTLA-4 or PD-1 blocking antibodies, whether they were given systemically (most effective) or locally (less effective), systemic administration of TLR9 agonist showed no efficacy alone or in combination with checkpoint blockade. Mechanistically, the addition of checkpoint blockade improves intratumoral ratios of CD8 $\mathrm{T}$ cells relative to suppressive stroma in the uninjected lesion and improves functional attributes of these critical effectors of anti-tumor immunity. Finally, we show that by combining both an enhanced potency TLR9 agonist (MGN1703 [10]) and a depletion-optimized CTLA-4 antibody (9D9-mIgG2a [11]), half of preimplanted parental B16-F10 melanoma can be cured.

\section{Materials and methods}

\section{Animals}

Male (6wk) C57BL/6 mice were purchased from the Jackson Laboratory (Bar Harbor, ME). All procedures were conducted in accordance with the guidelines established by the U.T. MD Anderson Cancer Center Institutional Animal Care and Use Committee.

\section{Cell lines and reagents}

B16-F10 melanoma and B16-Ova were obtained/created and cultured as described [12, 13].

\section{Therapeutic antibodies}

CTLA-4 (9H10 [Syrian Hamster Ig], $100 \mu \mathrm{g} /$ dose) and PD-1 (RMP1-14 [Rat IgG2a], $250 \mu \mathrm{g} /$ dose) antibodies were purchased from BioXCell or Leinco. CTLA-4 (9D9

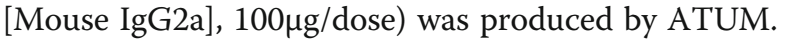

\section{TLR9 agonists}

ODN1826 was obtained from Invivogen, reconstituted in PBS and given either via intra-tumoral (local, 10 $\mu \mathrm{g}$ or $30 \mu \mathrm{g}$ in 50ul) or intra-peritoneal (systemic, $10 \mu \mathrm{g}$ or $30 \mu \mathrm{g}$ in 100ul) administration. MGN1703 was obtained from Mologen, diluted in PBS, and administered intratumorally at $30 \mu \mathrm{g}$ in $50 \mathrm{ul}$.

\section{Tumor therapy}

Mice were implanted s.c. with $1.5 \times 10^{5}$ B16-Ova or $2.5 \times 10^{4}$ B16-F10 cells on the flank as described $[12,13]$. For isolation of tumor infiltrating lymphocytes, tumors are implanted in 30\% Matrigel (Corning). On days 3, 6, and 9 mice received the indicated antibody and/or TLR 9 agonist i.p or intra-tumorally as indicated. B16 melanoma tumors leave a black spot at the injection site which is used to localize intra-tumoral injections when tumors are not yet palpable. Animals are followed and tumor growth tracked until tumors reach $1000 \mathrm{~mm}^{3}$ in size.

\section{Cell isolation}

Tumors were isolated, digested into single cell suspensions, and enriched for viable lymphocytes as previously described $[14,15]$.

\section{Flow cytometry analysis}

Tumor infiltrating lymphocytes were isolated by enzymatic digestion of tumor and enrichment over a Histopaque 1119 (Sigma) gradient. Samples were fixed using the Foxp3/Transcription Factor Staining Buffer Set (Thermo) and then stained with up to 12 antibodies at a time from Biolegend, BD Biosciences, and Thermo. Flow cytometry data was collected on an 18-color BD LSR II cytometer and analyzed in FlowJo (Treestar).

\section{Statistical analysis}

All statistics were calculated using Graphpad Prism Version 8 for Windows. Statistical significance was determined using the Mantel-Cox (Logrank) test for survival and ANOVA for tumor-infiltrating lymphocyte analysis. Graphs show mean \pm standard deviation unless otherwise indicated. $P$-values less than 0.05 were considered significant. 


\section{Results}

The combination of intra-tumoral ODN1826 and the antiCTLA-4 antibody $9 \mathrm{H} 10$ promotes rejection of bilateral B16-ova melanoma

We sought to test whether activation of TLR9 through intra-tumoral injection in the B16-Ova melanoma model could potentiate systemic, sterilizing anti-tumor immunity in conjunction with blockade of the $\mathrm{T}$ cell immune checkpoint receptor CTLA-4. C57BL/6 J mice were injected with $1.5 \times 10^{5}$ B16-Ova melanoma cells on the right and left flanks. The right flank tumor was then injected with $30 \mu \mathrm{g}$ of the TLR9 agonist ODN1826 or PBS on days 3, 6 and 9 with or without concordant injection of $100 \mu \mathrm{g}$ of the CTLA- 4 blocking antibody $9 \mathrm{H} 10$ systemically. The combination of TLR9 activation and CTLA-4 blockade cures $44 \%$ of mice of both the injected and uninjected tumors, while survival with either monotherapy is $10 \%$ or less (Fig. 1a). TLR9 injected tumors on the right flank largely resolve; however, resolution of the left flank tumor is only pronounced in combination with CTLA-4 blockade (Fig. 1b). These data illustrate therapeutic synergy of innate activation of the tumor stroma by the TLR9 agonist combined with regulatory $\mathrm{T}$ cell (Treg) depletion and effector $\mathrm{T}$ cell checkpoint blockade from the CTLA-4 antibody $9 \mathrm{H} 10$. In this setting, the
TLR9 agonist converts the injected tumor into an in situ vaccine and the checkpoint provides the conditioning of uninjected tumor sites and protection from attenuation necessary for the mobilized $\mathrm{T}$ cells to mediate effective abscopal responses. While this data demonstrates the high therapeutic potential of this combination in an immunogenic tumor setting, we sought to determine the optimal dose, schedule, and administration of this pair in the parental, poorly immunogenic B16-F10 model.

Intra-tumoral, but not systemic ODN1826 cooperates with either systemic CTLA-4 or PD-1 blockade to treat B16-F10 melanoma

Having shown the cooperative potential of CTLA-4 blockade and TLR9 activation, we sought to determine whether anti-PD-1 could substitute for anti-CTLA-4, whether TLR9 activation was most efficient locally or systemically, and whether both the checkpoint antibody and TLR9 agonist could be given locally to avoid systemic toxicities. We implanted $2.5 \times 10^{4}$ B16-F10 melanoma cells on the right and left flanks and treated mice

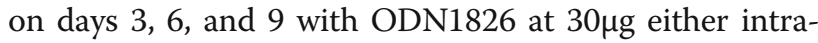
tumorally or systemically with or without concomitant anti-CTLA-4 (9H10) or anti-PD-1 either locally $(10 \mu \mathrm{g})$ or systemically $(100 \mu \mathrm{g} / 250 \mu \mathrm{g})$. Compared with B16-Ova,

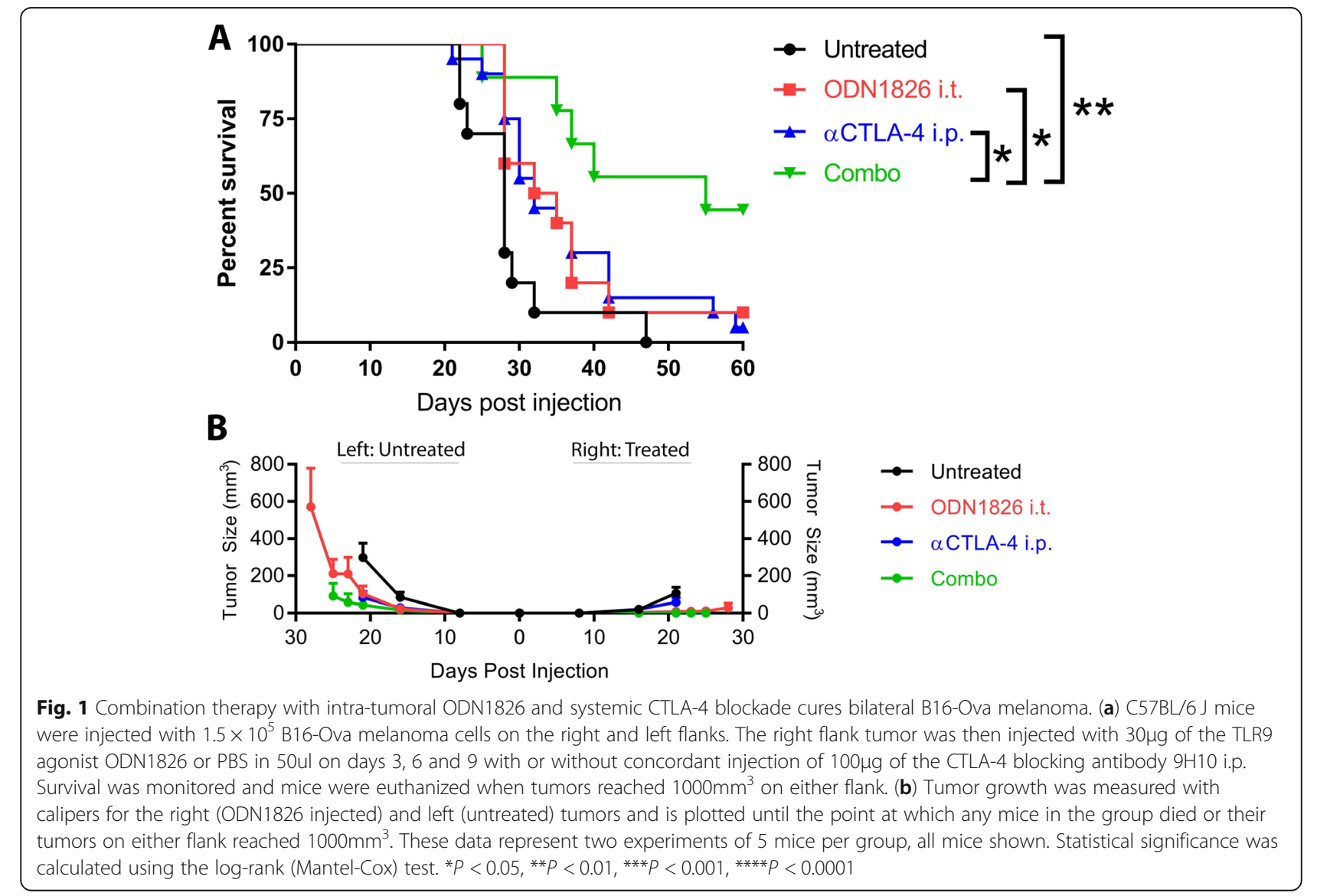


the non-immunogenic B16-F10 melanoma was significantly less responsive to monotherapy treatments; however, TLR9 agonist $(p=0.0054)$, anti-CTLA-4 $(p=$ $0.0125)$ and anti-PD-1 $(p=0.0283)$ all showed a modest capacity to extend survival (Fig. 2a). Combinations of ODN1826 with local anti-CTLA-4 $(p=0.014)$ or antiPD-1 ( $p=0.0053)$ was superior to control, but failed to show significant improvement versus the component therapies. Intra-tumoral TLR9 agonist readily elicited rejection of the injected lesion; however, there was no evidence of abscopal activity against the uninjected tumor on the opposite flank (Fig. 2b). Given the lack of potentiation of local TLR9 agonist activity by local checkpoint blockade against this poorly immunogenic melanoma, we explored the potential of systemic administration of these immunotherapies.

Systemic ODN1826, CTLA-4 blockade and PD-1 blockade were ineffective at extending survival in bilateral B16-F10 bearing animals. Both the TLR9 agonist and anti-CTLA-4 antibody lost all efficacy when administered systemically, in contrast to their activity when used locally in the right flank lesion (Fig. 2c, d). Given the lack of efficacy of systemic TLR9 agonist, we focused on combinations of local TLR9 agonist and systemic checkpoint blockade. In this context, intra-tumoral ODN1826 combined with systemic CTLA-4 blockade to extend survival versus control $(p=0.0016)$ and versus anti-CTLA-4 alone $(p=0.0094)$. Local TLR9 agonist with systemic PD-1 blockade also extended survival versus control $(p=0.0061)$, but only trended toward superiority to anti-PD-1 alone ( $p=0.06$, Gehan-BreslowWilcoxon test). Of note, this combination of local ODN1826 with systemic PD-1 blockade was also superior to local TLR9 agonist ( $p=0.011)$; however, the benefit of CTLA-4 blockade and TLR9 agonist over local ODN1826 alone did not reach significance (Fig. 2c, d). In order to understand the mechanisms underlying the differential efficacy across these combinations, we decided to assess each of their impacts on the lymphocyte infiltrate of the uninjected tumor.

\section{TLR9 activation with CTLA-4 or PD-1 blockade improves ratios of CD8 $T$ cells versus suppressive lymphocytes and myeloid cells}

While the intra-tumorally injected melanoma on the right flank almost always resolves, we wanted to assess changes in the immune infiltration of the unmanipulated left flank tumor to measure abscopal potential of each therapy. We established and treated bilateral B16-F10 tumors as described previously except that tumors were implanted in 30\% Matrigel (Corning) to facilitate recovery of infiltrating lymphocytes. At day 14 postimplantation, tumors were isolate, dispersed into single cells, enriched for viable lymphocytes over a Histopaque
1119 (Sigma) gradient, and then stained for analysis by flow cytometry. Intra-tumoral ratios of CD8 T cells versus $\mathrm{FoxP}^{+}$Tregs were significantly increased by the systemic CTLA-4 blockade alone or with intra-tumoral TLR9 agonist consistent with the known activity of this CTLA-4 antibody to deplete Tregs (Fig. 3a and Additional file 1: Figure S1). Despite this, the systemic CTLA-4 blockade and local TLR9 agonist combination trends toward significance over TLR9 alone $(p=0.059)$ and systemic anti-CTLA- $4(p=0.089)$ but did not reach significance. Notably, the combination of local TLR9 and local CTLA-4 also significantly improved CD8 to Treg ratios in the untreated lesion reflecting improved mobilization of CD8s at the injected lesion, which then traffic to the left flank tumor. We observed little benefit of the PD-1 combination in this regard. In contrast, ratios of CD8 $\mathrm{T}$ cells to myeloid-derived suppressor cells (MDSC) benefitted most from the combination of TLR9 agonist delivered to the right flank tumor combined with systemic PD-1 blockade (Fig. 3b). This combination was superior to anti-PD-1 alone; however, not in comparison to local TLR9 agonist alone. Again, local PD-1 blockade combined with TLR9 agonist also significantly improved the CD8 to MDSC ratio in the untreated lesion. There was also a significant improvement in the group receiving systemic CTLA-4 blockade with TLR9 intratumorally perhaps reflecting more efficient CD8 mobilization from the untreated tumor Across the analysis of these tumor infiltrating lymphocyte ratios, it was generally only the combinations of local TLR9 agonist and checkpoint blockade which showed significant benefit over untreated. We speculate that the more limited capacity to show statistically relevant benefit over the component monotherapies was likely a product of the limited overall efficacy of these interventions against the parental B16 melanoma.

Consistent with the known of effect of PD-1 blockade to restore suppressed $\mathrm{T}$ cell effector function, the most significant elevation of CD8 $\mathrm{T}$ cell Granzyme $\mathrm{B}$ frequency was observed in mice receiving the combination of local TLR9 agonist and systemic PD-1 blockade (Fig. 3c). Also, local PD-1 blockade and local TLR9 agonist significantly improved Granzyme B, as did systemic CTLA-4 blockade and local ODN1826. As for the effector to suppressor ratios, the combination therapies did not elevate Granzyme B frequency significantly beyond the monotherapies; however, none of the monotherapies conferred significant benefit versus untreated. In terms of proliferation, TLR9 agonist clearly promoted expansion of $\mathrm{T}$ cells which trafficked to the untreated lesion (Fig. 3d).

In summary, analysis of the infiltrate of the uninjected melanoma reveals that intra-tumoral TLR9 agonist therapy may mobilize CD8 $\mathrm{T}$ cells that traffic to the distal 


\section{A Survival: Local TLR9 \& checkpoint blockade}

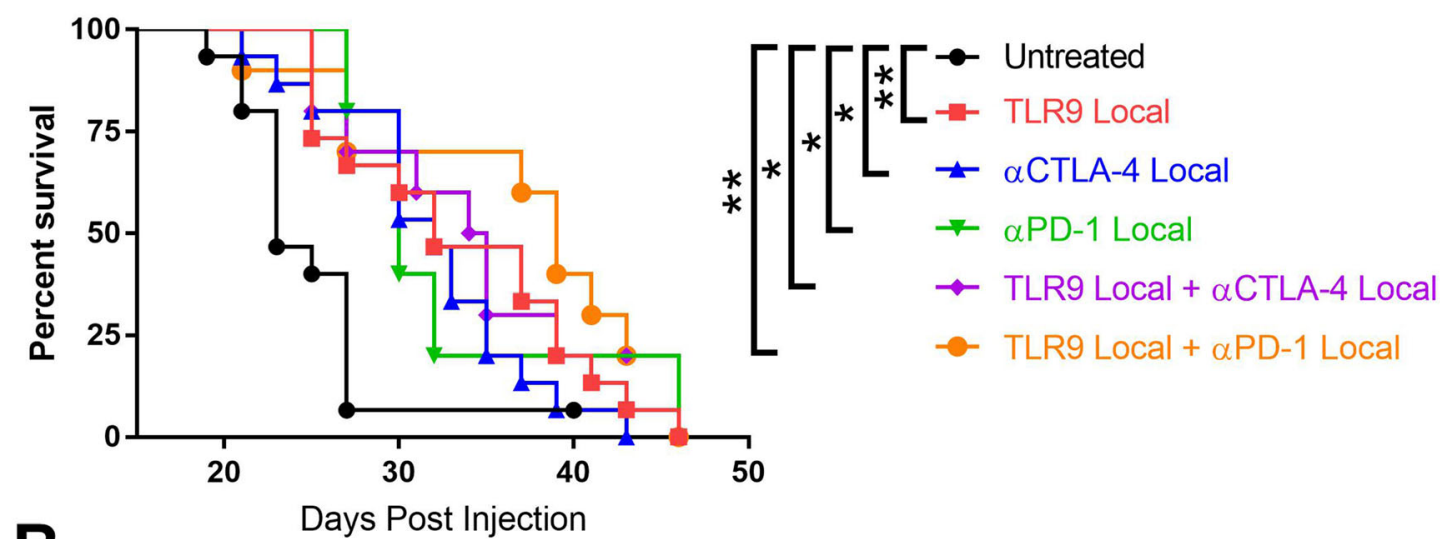

\section{B Local TLR9 \& local checkpoint blockade}

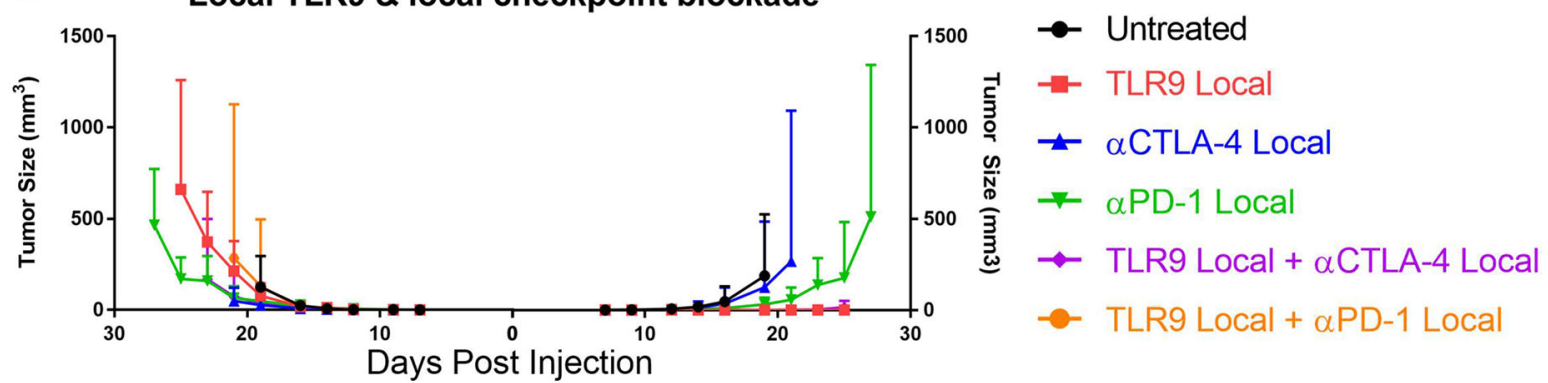

C Survival: Systemic TLR9 \& checkpoint blockade
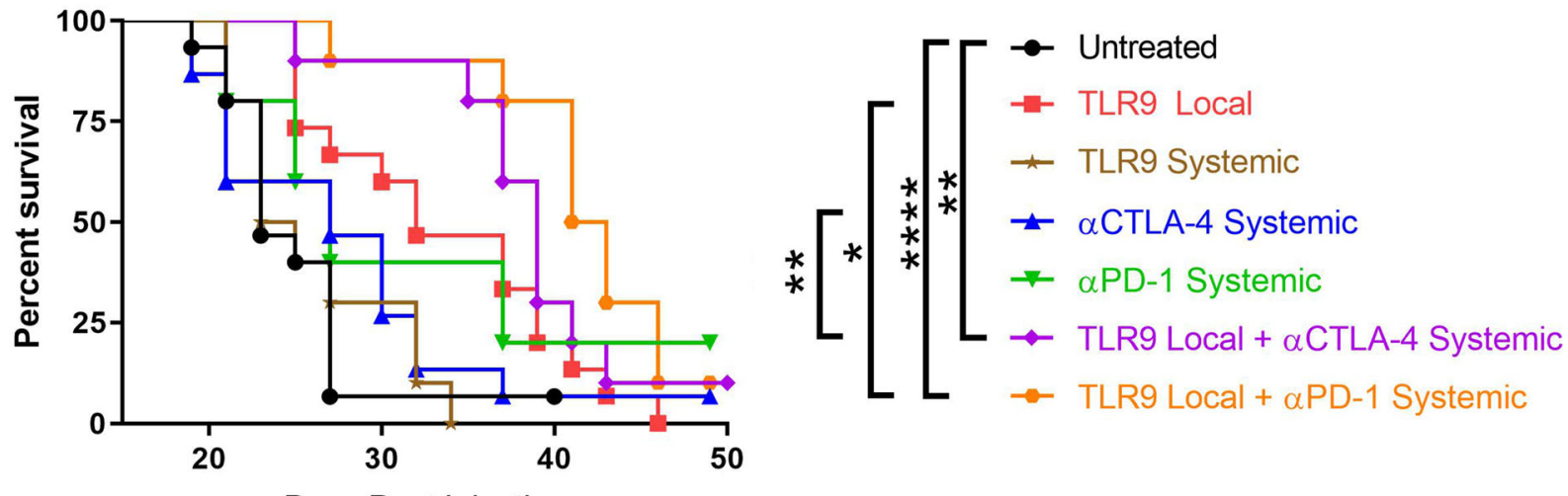

D Local TLR9 \& systemic checkpoint blockade

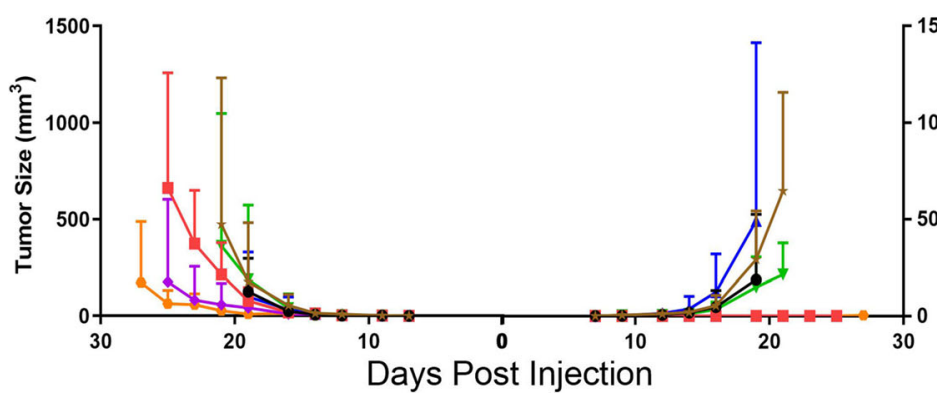

$\rightarrow$ Untreated

-- TLR9 Local

* TLR9 Systemic

$\leftarrow \alpha$ CTLA-4 Systemic

$\rightarrow$ aPD-1 Systemic

$\leadsto$ TLR9 Local $+\alpha$ CTLA-4 Systemic

$\rightarrow$ TLR9 Local + $\alpha$ PD-1 Systemic

Fig. 2 (See legend on next page.) 
(See figure on previous page.)

Fig. 2 Local but not systemic TLR9 activation cooperates with either CTLA-4 or PD-1 blockade to treat B16-F10 melanoma. C57BL/6 J mice were injected with $2.5 \times 10^{4}$ B16-F10 melanoma cells on the right and left flanks. (a) The right flank tumor was then injected with 30 $\mu \mathrm{g}$ of the TLR9

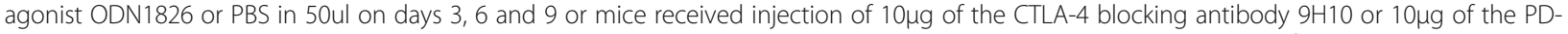
1 antibody RMP1-14 intra-tumorally. Survival was monitored and mice were euthanized when tumors reached $1000 \mathrm{~mm}^{3}$. (b) Tumor growth was measured with calipers for the right (ODN1826 injected) and left (untreated) tumors. (c) As above except that mice received injection of 100 $\mathrm{\mu g}$ of the CTLA-4 blocking antibody $9 \mathrm{H} 10$ or 250 $\mathrm{\mu g}$ of the PD-1 antibody RMP1-14 i.p. Survival was monitored and mice were euthanized when tumors reached $1000 \mathrm{~mm}^{3}$. (d) Tumor growth was measured with calipers for the right (ODN1826 injected) and left (untreated) tumors. These data represent one (antibody monotherapies) to two (all combinations) independent experiments of 5-10 mice per group, all mice are shown. Statistical significance was calculated using the log-rank (Mantel-Cox) test. ${ }^{*} P<0.05,{ }^{* *} P<0.01,{ }^{* * *} P<0.001,{ }^{* * * *} P<0.0001$
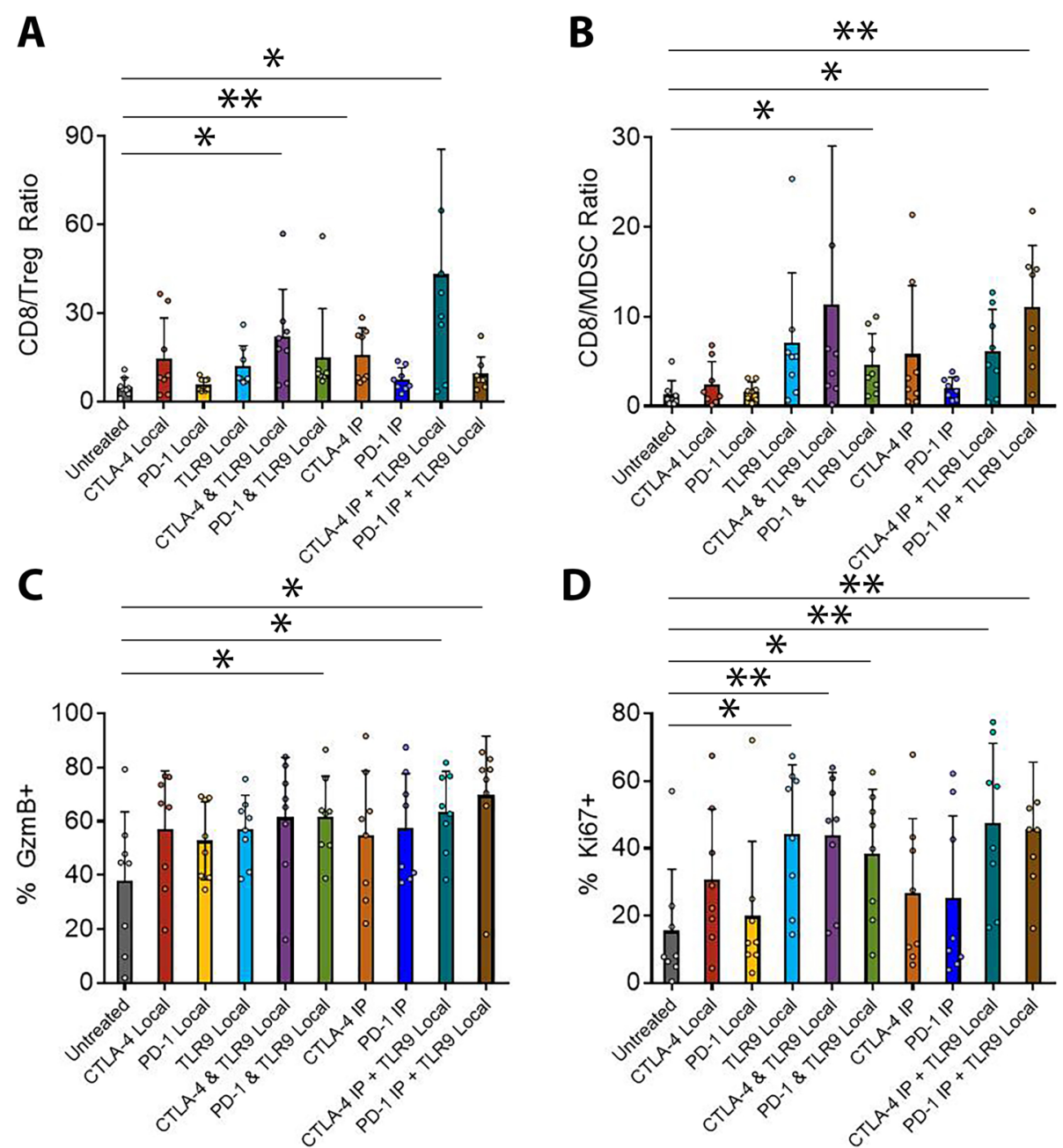

Fig. 3 CTLA-4 and PD-1 blockade each potentiate intra-tumoral TLR9 activation through distinct mechanisms. (A) C57BL/6 J mice were injected with $1.5 \times 10^{5}$ B16-Ova melanoma cells on the right and left flanks. The right flank tumor was then injected with $30 \mu \mathrm{g}$ of the TLR9 agonist

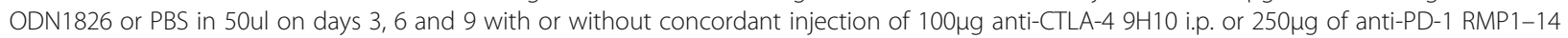
i.p. or $10 \mu \mathrm{g}$ of either antibody intra-tumorally. Mice were euthanized on day 14, tumors treated with Collagenase H (Sigma) and DNase (Roche) to produce single cell suspensions, and tumor infiltrating lymphocytes enriched via separation over a Histopaque 1119 (Sigma) density gradient ( $n=1$ experiment with 8 mice / group). Cells were fixed with the FoxP3 fixation kit (Thermo), stained with antibodies and analyzed by flow cytometry. (a) The ratios of intra-tumoral CD8 T cells versus FoxP3+ Treg and (b) versus CD11 b GR-1 $1^{+}$MDSC were determined. (c) For intratumoral CD8 T cells, the percentage expressing Granzyme B and (d) Ki67 was also measured. Statistical significance was calculated using the student's t-test. ${ }^{*} P<0.05,{ }^{* *} P<0.01,{ }^{* * *} P<0.001,{ }^{* * *} P<0.0001$ 
lesion and benefit from anti-CTLA-4 mediated Treg depletion or enhanced expansion relative to suppressive myeloid stroma mediated by anti-PD-1. Although local checkpoint blockade with local ODN1826 failed to statistically improve overall survival versus the component monotherapies, it did improve the immune infiltrate in the distal tumor, albeit to a lesser degree than systemic checkpoint blockade. CD8 T cell effector function was most significantly improved by the combination of local TLR9 activation and PD-1 blockade in a setting where none of the component monotherapies impacted Granzyme B frequency. Local TLR9 agonist alone or in combination with either checkpoint-blocking antibody significantly increased frequencies of actively proliferating CD8 $\mathrm{T}$ cells that trafficked to the untreated lesion on the opposite flank. Given these observations, we sought to determine whether a more potent TLR9 agonist and a more efficient Treg depleting CTLA-4 antibody could further augment the efficacy of this combination therapy.

\section{Intra-tumoral MGN1703 combined with systemic 9D9- IgG2a anti-CTLA-4 antibody cures poorly immunogenic B16-F10 melanoma}

MGN1703 is a potent, clinical-stage TLR9 agonist which has recently been described [10]. The mouse anti-mouse CTLA-4 antibody 9D9 is an exceptionally efficient depleter of Tregs when expressed with the mouse IgG2a isotype, and is not subject to antibody-mediated neutralization over repeated administration as is the hamster-derived $9 \mathrm{H} 10$ clone used previously [11]. C57BL/6 J mice were injected with $2.5 \times 10^{4}$ B16-F10 melanoma cells on the right and left flanks. The right flank tumor was then injected with $30 \mu \mathrm{g}$ of the TLR9 agonist MGN1703 or PBS on days 3, 6 and 9 with or without concordant injection of $100 \mu \mathrm{g}$ of the CTLA-4 antibody 9D9-mIgG2a systemically. Whereas no animals survived in the prior study with ODN1826 and 9H10,
50\% of MGN1703 and 9D9-mIgG2a treated mice are cured of bilateral B16-F10 (Fig. 4). The higher activity CTLA-4 antibody accounts for much of this improvement with $13 \%$ survival as a monotherapy; however, there appears to be clear synergy with the more potent TLR9 agonist as well $(p=0.04)$. While the improved potency of MGN1703 for TLR9 activation has been reported previously [10], it is impossible for us to preclude a similar benefit with the ODN1826 combination in this context. These data demonstrate that highly significant improvements in survival and cure rate for bilateral melanoma can be achieved by using higher potency TLR9 and CTLA-4 blocking / depleting antibodies.

\section{Discussion}

In this study, we sought to answer a series of questions designed to inform optimal design of the growing number of clinical trials seeking to combine activation of innate immunity through TLR engagement with augmentation of the mobilized $\mathrm{T}$ cell response through checkpoint antibody administration. Administration of the TLR9 agonist ODN1826 was consistently effective at inducing rejection of the injected lesion and in creating advantageous CD8 to MDSC rations in distal lesions when given intra-tumorally. In contrast, there was no therapeutic benefit to systemic TLR9 agonist alone or in combination with checkpoint blockade likely reflecting a lack of specific immune activation in the tumor microenvironment. Both CTLA-4 and PD-1 blockade could potentiate in situ vaccination through intra-tumoral TLR9 activation with systemic administration, while local administration only effected sub-therapeutic improvements to the uninjected tumor. In the clinic, local administration would engender no appreciable systemic toxicity and prior publications have suggested that intratumoral administration of high order combinations of checkpoint antagonists and co-stimulatory agonists may

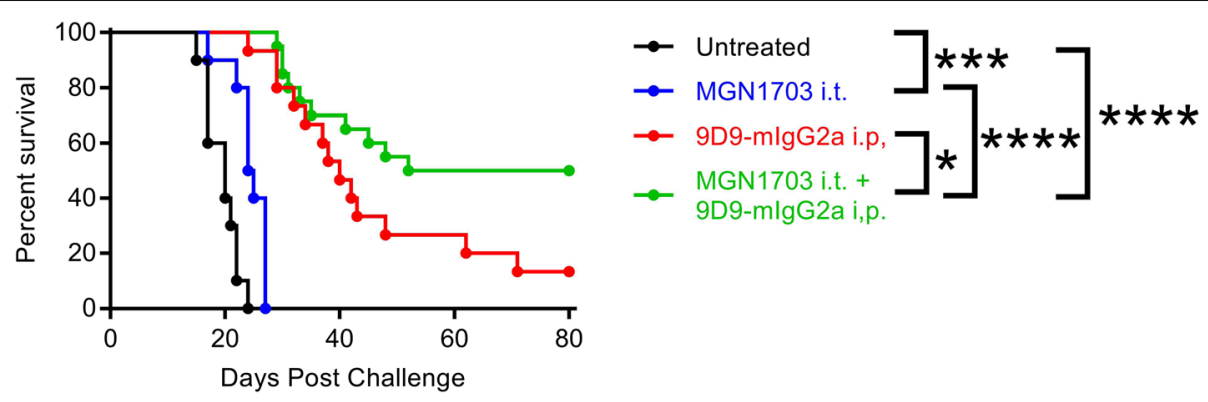

Fig. 4 Intra-tumoral MGN1703 combined with systemic 9D9-IgG2a anti-CTLA-4 antibody cures poorly immunogenic B16-F10 melanoma. C57BL/6 $\mathrm{J}$ mice were injected with $2.5 \times 10^{4} \mathrm{~B} 16-\mathrm{F} 10$ melanoma cells on the right and left flanks. The right flank tumor was then injected with $30 \mu \mathrm{g}$ of the

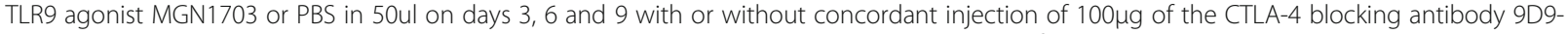
mlgG2a i.p. Survival was monitored and mice were euthanized when tumors reached $1000 \mathrm{~mm}^{3}(n=2$ independent experiments of 5-10 mice / group, all shown). Statistical significance was calculated using the log-rank (Mantel-Cox) test. ${ }^{*} P<0.05,{ }^{* *} P<0.01,{ }^{* * *} P<0.001,{ }^{* * * *} P<0.0001$ 
be significantly more effective than the results we obtained here with monotherapy combinations [15].

Mechanistically, improved CD8 to Treg ratios in the uninjected tumor appeared critical for conditioning an environment in which $\mathrm{T}$ cell mobilized from the TLR9treated lesion could flourish. Although current clinical CTLA-4 antibodies do not efficiently deplete Tregs from solid tumors, development of 2nd generation CTLA-4 antibodies for patients that can kill Tregs either systemically or specifically in the tumor microenvironment are nearing the clinic. Our data would suggest that such drugs may powerfully synergize with TLR agonists to more effectively potentiate abscopal anti-tumor immunity. PD-1 blockade, which is the prevalent immunotherapy in the clinic, also showed equivalent combination potential to CTLA-4 blockade when paired with intratumoral TLR9 agonist. Interestingly, the CTLA-4 combination created enhanced CD8 to Treg ratios in the distal tumor, whereas the PD-1 combination yielded improved ratios of $\mathrm{CD} 8$ relative to suppressive myeloid stroma. This suggests that the combination of CTLA-4 and PD-1 blockade in this context may synergize in improving therapeutic outcomes in this setting.

It remained unclear to what extent the potency of the component TLR9 agonist and CTLA-4 antibody acted together to set a threshold of efficacy for this combination therapy. We show that a more potent TLR9 agonist (i.e. MGN1703 versus ODN1826) combined with a more potent CTLA-4 antibody (i.e. 9D9-mIgG2a versus 9H10) very significantly increased the therapeutic potential of this combination against bilateral, poorly immunogenic B16-F10 melanoma (0\% versus $50 \%$ tumorfree survival). In the clinic this suggests that more powerful innate agonists can deliver greater therapeutic benefit and that the advent of anti-human CTLA- 4 antibodies with the capacity to deplete Tregs, particularly in a tumor-selective fashion, could profoundly improve outcomes against poorly immunogenic cancers.

\section{Supplementary information}

Supplementary information accompanies this paper at https://doi.org/10. 1186/s40425-019-0811-x.

Additional file 1. Flow cytometry gating strategy for tumor infiltrating lymphocyte analysis.

\section{Abbreviations}

CTLA-4: Cytotoxic T-lymphocyte-associated antigen 4; MDSC: Myeloidderived suppressor cell; ODN: Oligodeoxynucleotide; Ova: Ovalbumin; PD1: Programmed Cell Death 1; TAM: Tumor associated macrophage; TLR: Tolllike Receptor

\section{Authors' contributions}

MJR, BM, DSH, and MAC participated in the design and/or interpretation of experiments. MJR, BM, and AL, MAC participated in the acquisition and/or analysis of experimental data. DSH, and MAC provided supervisory support. MJR, BM, DSH, AL and MAC participated in writing and/or revising the manuscript. All authors read and approved the final manuscript.

Funding

Not applicable.

\section{Availability of data and materials}

The datasets used and/or analyzed during the current study available from the corresponding author on request.

\section{Ethics approval}

All animal studies were approved by the University of Texas MD Anderson Cancer Center IACUC under protocol 00001378-RN01.

Consent for publication

Not Applicable.

\section{Competing interests}

MC is a consultant for Merck, Inc. and the Founder and Chairman of the Board of Immunogenesis, Inc. The remaining authors declare no potential conflicts of interest.

\section{Author details}

${ }^{1}$ Department of Medicine, The University of Virginia, Charlottesville, VA 22903, USA. ${ }^{2}$ Department of Immunology, The University of Texas MD Anderson Cancer Center, Houston, TX 77030, USA. ${ }^{3}$ Department of Investigational Cancer Therapeutics, The University of Texas MD Anderson Cancer Center, Houston, TX 77030, USA. ${ }^{4}$ The University of Texas MD Anderson UTHealth Graduate School of Biomedical Sciences; Immunology Program, Houston, TX 77030, USA

\section{Received: 12 August 2019 Accepted: 6 November 2019}

\section{References}

1. Lu H. TLR agonists for Cancer immunotherapy: tipping the balance between the immune stimulatory and inhibitory effects. Front Immunol. 2014;5:83.

2. Krieg AM, Yi AK, Matson S, Waldschmidt TJ, Bishop GA, Teasdale R, et al. CpG motifs in bacterial DNA trigger direct B-cell activation. Nature. 1995; 374(6522):546-9.

3. Sato-Kaneko F, Yao S, Ahmadi A, Zhang SS, Hosoya T, Kaneda MM, et al. Combination immunotherapy with TLR agonists and checkpoint inhibitors suppresses head and neck cancer. JCI Insight. 2017:2(18).

4. Davila E, Kennedy R, Celis E. Generation of antitumor immunity by cytotoxic T lymphocyte epitope peptide vaccination, CpG-oligodeoxynucleotide adjuvant, and CTLA-4 blockade. Cancer Res. 2003;63(12):3281-8.

5. Mangsbo SM, Sandin LC, Anger K, Korman AJ, Loskog A, Totterman TH. Enhanced tumor eradication by combining CTLA-4 or PD-1 blockade with CpG therapy. J Immunother. 2010;33(3):225-35.

6. Sagiv-Barfi I, Czerwinski DK, Levy S, Alam IS, Mayer AT, Gambhir SS, et al. Eradication of spontaneous malignancy by local immunotherapy. Sci Transl Med. 2018;10(426)

7. Brody JD, Ai WZ, Czerwinski DK, Torchia JA, Levy M, Advani RH, et al. In situ vaccination with a TLR9 agonist induces systemic lymphoma regression: a phase I/II study. J Clin Oncol. 2010;28(28):4324-32.

8. Marabelle A, Kohrt H, Sagiv-Barfi I, Ajami B, Axtell RC, Zhou G, et al. Depleting tumor-specific Tregs at a single site eradicates disseminated tumors. J Clin Invest. 2013;123(6):2447-63.

9. Davis HL, Weeratna R, Waldschmidt TJ, Tygrett L, Schorr J, Krieg AM. CpG DNA is a potent enhancer of specific immunity in mice immunized with recombinant hepatitis B surface antigen. J Immunol. 1998;160(2):870-6.

10. Kapp K, Volz B, Oswald D, Wittig B, Baumann M, Schmidt M. Beneficial modulation of the tumor microenvironment and generation of anti-tumor responses by TLR9 agonist lefitolimod alone and in combination with checkpoint inhibitors. Oncolmmunology. 2019:8:12. https://doi.org/10.1080/ 2162402X.2019.1659096. 
11. Selby MJ, Engelhardt JJ, Quigley M, Henning KA, Chen T, Srinivasan M, et al. Anti-CTLA-4 antibodies of IgG2a isotype enhance antitumor activity through reduction of intratumoral regulatory T cells. Cancer Immunol Res. 2013;1(1): 32-42.

12. Curran MA, Allison JP. Tumor vaccines expressing Flt3 ligand synergize with CTLA-4 blockade to reject Preimplanted tumors. Cancer Res. 2009;69(19): 7747-55.

13. Curran MA, Kim M, Montalvo W, Al-Shamkhani A, Allison JP. Combination CTLA-4 blockade and 4-1BB activation enhances tumor rejection by increasing T-cell infiltration, proliferation, and cytokine production. PLoS One. 2011;6(4):e19499.

14. Jayaprakash P, Ai M, Liu A, Budhani P, Bartkowiak T, Sheng J, et al. Targeted hypoxia reduction restores $\mathrm{T}$ cell infiltration and sensitizes prostate cancer to immunotherapy. J Clin Invest. 2018.

15. Ager CR, Reilley MJ, Nicholas C, Bartkowiak T, Jaiswal AR, Curran MA. Intratumoral STING activation with T-cell checkpoint modulation generates systemic antitumor immunity. Cancer Immunol Res. 2017;5(8):676-84.

\section{Publisher's Note}

Springer Nature remains neutral with regard to jurisdictional claims in published maps and institutional affiliations.

Ready to submit your research? Choose BMC and benefit from:

- fast, convenient online submission

- thorough peer review by experienced researchers in your field

- rapid publication on acceptance

- support for research data, including large and complex data types

- gold Open Access which fosters wider collaboration and increased citations

- maximum visibility for your research: over $100 \mathrm{M}$ website views per year

At $\mathrm{BMC}$, research is always in progress.

Learn more biomedcentral.com/submissions 